\title{
Association Between Exposure to Nonactionable Physiologic Monitor Alarms and Response Time in a Children's Hospital
}

\author{
Christopher P. Bonafide, MD, MSCE ${ }^{1,2,3,4 *}$, Richard Lin, MD ${ }^{5,6}$, Miriam Zander, BA ${ }^{1}$, Christian Sarkis Graham, BA ${ }^{1}$, \\ Christine W. Paine, MPH${ }^{1}$, Whitney Rock, MS ${ }^{7}$, Andrew Rich, BS ${ }^{7}$, Kathryn E. Roberts, RN, MSN , Margaret Fortino, RN, MSN², \\ Vinay M. Nadkarni, MD, MS ${ }^{5,6}$, A. Russell Localio, PhD $^{10}$, Ron Keren, MD, MPH ${ }^{1,2,3,4}$
}

\begin{abstract}
'Division of General Pediatrics, The Children's Hospital of Philadelphia, Philadelphia, Pennsylvania; 'Department of Pediatrics, Perelman School of Medicine at the University of Pennsylvania, Philadelphia, Pennsylvania; ${ }^{3}$ Center for Pediatric Clinical Effectiveness, The Children's Hospital of Philadelphia, Philadelphia, Pennsylvania; ${ }^{4}$ Leonard Davis Institute of Health Economics, University of Pennsylvania, Philadelphia, Pennsylvania; ${ }^{5}$ Department of Anesthesiology and Critical Care Medicine, The Children's Hospital of Philadelphia, Philadelphia, Pennsylvania; ${ }^{6}$ Department of Anesthesiology and Critical Care, Perelman School of Medicine at the University of Pennsylvania, Philadelphia, Pennsylvania; ${ }^{7}$ Department of Biomedical Engineering, The Children's Hospital of Philadelphia, Philadelphia, Pennsylvania; ${ }^{8}$ Department of Nursing, The Children's Hospital of Philadelphia, Philadelphia, Pennsylvania; ${ }^{9}$ Department of Nursing, Hospital of the University of Pennsylvania, Philadelphia, Pennsylvania; ${ }^{10}$ Department of Biostatistics and Epidemiology, Perelman School of Medicine at the University of Pennsylvania, Philadelphia, Pennsylvania.
\end{abstract}

BACKGROUND: Alarm fatigue is reported to be a major threat to patient safety, yet little empirical data support its existence in the hospital.

OBJECTIVE: To determine if nurses exposed to high rates of nonactionable physiologic monitor alarms respond more slowly to subsequent alarms that could represent lifethreatening conditions.

DESIGN: Observational study using video.

SETTING: Freestanding children's hospital.

PATIENTS: Pediatric intensive care unit (PICU) patients requiring inotropic support and/or mechanical ventilation, and medical ward patients.

INTERVENTION: None.

MEASUREMENTS: Actionable alarms were defined as correctly identifying physiologic status and warranting clinical intervention or consultation. We measured response time to alarms occurring while there were no clinicians in the patient's room. We evaluated the association between the number of nonactionable alarms the patient had in the preceding 120 minutes (categorized as $0-29,30-79$, or $80+$ alarms) and response time to subsequent alarms in the same patient using a log-rank test that accounts for withinnurse clustering.

RESULTS: We observed 36 nurses for 210 hours with 5070 alarms; $87.1 \%$ of PICU and $99.0 \%$ of ward clinical alarms were nonactionable. Kaplan-Meier plots showed incremental increases in response time as the number of nonactionable alarms in the preceding 120 minutes increased (logrank test stratified by nurse $P<0.001$ in PICU, $P=0.009$ in the ward).

CONCLUSIONS: Most alarms were nonactionable, and response time increased as nonactionable alarm exposure increased. Alarm fatigue could explain these findings. Future studies should evaluate the simultaneous influence of workload and other factors that can impact response time. Journal of Hospital Medicine 2015;10:345-351. (C) 2015 Society of Hospital Medicine
Hospital physiologic monitors can alert clinicians to early signs of physiologic deterioration, and thus have great potential to save lives. However, monitors generate frequent alarms, ${ }^{1-8}$ and most are not relevant to the patient's safety (over $90 \%$ of pediatric intensive care unit (PICU) $)^{1,2}$ and over $70 \%$ of adult intensive care alarms). ${ }^{5,6}$ In psychology experiments, humans rapidly learn to ignore or respond more slowly to alarms when exposed to high false-alarm rates, exhibiting "alarm fatigue." 9,10 In 2013, The Joint Commis-

\footnotetext{
*Address for correspondence and reprint requests: Christopher $\mathrm{P}$ Bonafide, MD, The Children's Hospital of Philadelphia, 34th St. and Civic Center Blvd., Suite 12NW80, Philadelphia, PA 19104; Telephone: 267-426-2901; E-mail: bonafide@email.chop.edu

Additional Supporting Information may be found in the online version of this article.

Received: October 5, 2014; Revised: November 25, 2014; Accepted: December 7, 2014

2015 Society of Hospital Medicine DOI 10.1002/jhm.2331

Published online in Wiley Online Library (Wileyonlinelibrary.com).
}

sion named alarm fatigue the most common contributing factor to alarm-related sentinel events in hospitals. ${ }^{11,12}$

Although alarm fatigue has been implicated as a major threat to patient safety, little empirical data support its existence in hospitals. In this study, we aimed to determine if there was an association between nurses' recent exposure to nonactionable physiologic monitor alarms and their response time to future alarms for the same patients. This exploratory work was designed to inform future research in this area, acknowledging that the sample size would be too small for multivariable modeling.

\section{METHODS \\ Study Definitions}

The alarm classification scheme is shown in Figure 1. Note that, for clarity, we have intentionally avoided using the terms "true" and "false" alarms because their interpretations vary across studies and can be misleading. 


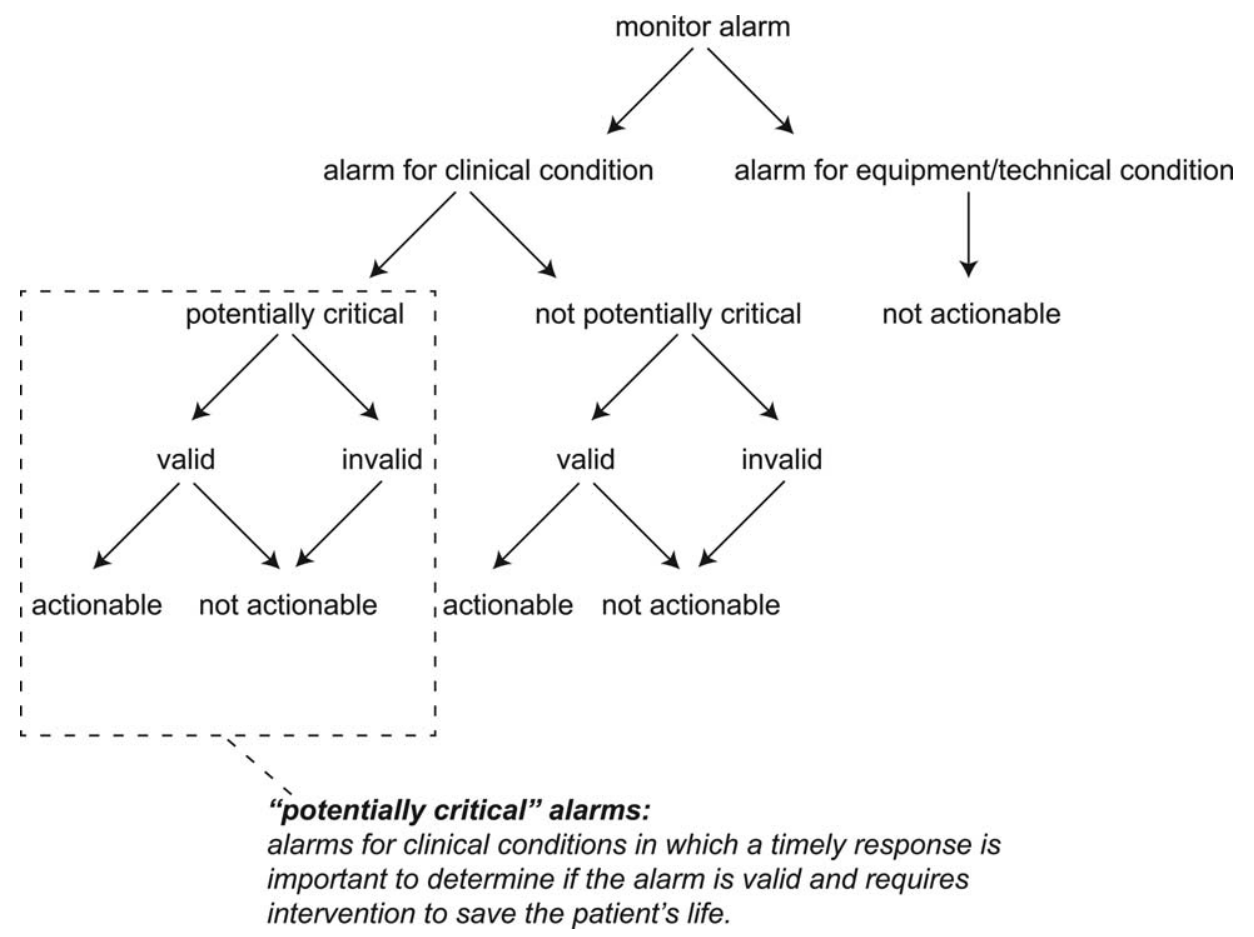

FIG. 1. Alarm classification scheme.

TABLE 1. Characteristics of the 2,445 Alarms for Clinical Conditions

\begin{tabular}{|c|c|c|c|c|c|c|c|c|}
\hline \multirow[b]{2}{*}{ Alarm type } & \multicolumn{4}{|c|}{ PICU } & \multicolumn{4}{|c|}{ Ward } \\
\hline & No. & $\%$ of Total & $\%$ Valid & $\%$ Actionable & No. & $\%$ of Total & $\%$ Valid & $\%$ Actionable \\
\hline Oxygen saturation & 197 & 19.4 & 82.7 & 38.6 & 590 & 41.2 & 24.4 & 1.9 \\
\hline Heart rate & 194 & 19.1 & 95.4 & 1.0 & 266 & 18.6 & 87.2 & 0.0 \\
\hline Respiratory rate & 229 & 22.6 & 80.8 & 13.5 & 316 & 22.1 & 48.1 & 1.0 \\
\hline Blood pressure & 259 & 25.5 & 83.8 & 5.8 & 11 & 0.8 & 72.7 & 0.0 \\
\hline Critical arrhythmia & 1 & 0.1 & 0.0 & 0.0 & 4 & 0.3 & 0.0 & 0.0 \\
\hline Noncritical arrhythmia & 71 & 7.0 & 2.8 & 0.0 & 244 & 17.1 & 8.6 & 0.0 \\
\hline Central venous pressure & 49 & 4.8 & 0.0 & 0.0 & 0 & 0.0 & $N / A$ & $N / A$ \\
\hline Exhaled carbon dioxide & 14 & 1.4 & 92.9 & 50.0 & 0 & 0.0 & N/A & N/A \\
\hline Total & 1014 & 100.0 & 75.6 & 12.9 & 1,431 & 100.0 & 38.9 & 1.0 \\
\hline
\end{tabular}

NOTE: Abbreviations: N/A, not applicable; PICU, pediatric intensive care unit.

\section{Potentially Critical Alarm}

A potentially critical alarm is any alarm for a clinical condition for which a timely response is important to determine if the alarm requires intervention to save the patient's life. This is based on the alarm type alone, including alarms for life-threatening arrhythmias such as asystole and ventricular tachycardia, as well as alarms for vital signs outside the set limits. Supporting Table 1 in the online version of this article lists the breakdown of alarm types that we defined a priori as potentially and not potentially critical.

\section{Valid Alarm}

A valid alarm is any alarm that correctly identifies the physiologic status of the patient. Validity was based on waveform quality, lead signal strength indicators, and artifact conditions, referencing each monitor's operator's manual.

\section{Actionable Alarm}

An actionable alarm is any valid alarm for a clinical condition that either: (1) leads to a clinical intervention; (2) leads to a consultation with another clinician at the bedside (and thus visible on camera); or (3) is a situation that should have led to intervention or consultation, but the alarm was unwitnessed or misinterpreted by the staff at the bedside.

\section{Nonactionable Alarm}

An unactionable alarm is any alarm that does not meet the actionable definition above, including invalid alarms such as those caused by motion artifact, 
equipment/technical alarms, and alarms that are valid but nonactionable (nuisance alarms). ${ }^{13}$

\section{Response Time}

The response time is the time elapsed from when the alarm fired at the bedside to when the nurse entered the room or peered through a window or door, measured in seconds.

\section{Setting and Subjects}

We performed this study between August 2012 and July 2013 at a freestanding children's hospital. We evaluated nurses caring for 2 populations: (1) PICU patients with heart and/or lung failure (requiring inotropic support and/or invasive mechanical ventilation), and (2) medical patients on a general inpatient ward. Nurses caring for heart and/or lung failure patients in the PICU typically were assigned 1 to 2 total patients. Nurses on the medical ward typically were assigned 2 to 4 patients. We identified subjects from the population of nurses caring for eligible patients with parents available to provide in-person consent in each setting. Our primary interest was to evaluate the association between nonactionable alarms and response time, and not to study the epidemiology of alarms in a random sample. Therefore, when alarm data were available prior to screening, we first approached nurses caring for patients in the top $25 \%$ of alarm rates for that unit over the preceding 4 hours. We identified preceding alarm rates using BedMasterEx (Excel Medical Electronics, Jupiter, FL).

\section{Human Subjects Protection}

This study was approved by the institutional review board of The Children's Hospital of Philadelphia. We obtained written in-person consent from the patient's parent and the nurse subject. We obtained a Certificate of Confidentiality from the National Institutes of Health to further protect study participants. ${ }^{14}$

\section{Monitoring Equipment}

All patients in the PICU were monitored continuously using General Electric (GE) (Fairfield, CT) solar devices. All bed spaces on the wards include GE Dash monitors that are used if ordered. On the ward we studied, $30 \%$ to $50 \%$ of patients are typically monitored at any given time. In addition to alarming at the bedside, most clinical alarms also generated a text message sent to the nurse's wireless phone listing the room number and the word "monitor." Messages did not provide any clinical information about the alarm or patient's status. There were no technicians reviewing alarms centrally.

Physicians used an order set to order monitoring, selecting 1 of 4 available preconfigured profiles: infant $<6$ months, infant 6 months to 1 year, child, and adult. The parameters for each age group are in Supporting Figure 1, available in the online version of this article. A physician order is required for a nurse to change the parameters. Participating in the study did not affect this workflow.

\section{Primary Outcome}

The primary outcome was the nurse's response time to potentially critical monitor alarms that occurred while neither they nor any other clinicians were in the patient's room.

\section{Primary Exposure and Alarm Classification}

The primary exposure was the number of nonactionable alarms in the same patient over the preceding 120 minutes (rolling and updated each minute). The alarm classification scheme is shown in Figure 1.

Due to technical limitations with obtaining timestamped alarm data from the different ventilators in use during the study period, we were unable to identify the causes of all ventilator alarms. Therefore, we included ventilator alarms that did not lead to clinical interventions as nonactionable alarm exposures, but we did not evaluate the response time to any ventilator alarms.

\section{Data Collection}

We combined video recordings with monitor timestamp data to evaluate the association between nonactionable alarms and the nurse's response time. Our detailed video recording and annotation methods have been published separately. ${ }^{15}$ Briefly, we mounted up to 6 small video cameras in patients' rooms and recorded up to 6 hours per session. The cameras captured the monitor display, a wide view of the room, a close-up view of the patient, and all windows and doors through which staff could visually assess the patient without entering the room.

\section{Video Processing, Review, and Annotation}

The first 5 video sessions were reviewed in a group training setting. Research assistants received instruction on how to determine alarm validity and actionability in accordance with the study definitions. Following the training period, the review workflow was as follows. First, a research assistant entered basic information and a preliminary assessment of the alarm's clinical validity and actionability into a REDCap (Research Electronic Data Capture; Vanderbilt University, Nashville, TN) database. ${ }^{16}$ Later, a physician investigator secondarily reviewed all alarms and confirmed the assessments of the research assistants or, when disagreements occurred, discussed and reconciled the database. Alarms that remained unresolved after secondary review were flagged for review with an additional physician or nurse investigator in a team meeting.

\section{Data Analysis}

We summarized the patient and nurse subjects, the distributions of alarms, and the response times to potentially critical monitor alarms that occurred while 
neither the nurse nor any other clinicians were in the patient's room. We explored the data using plots of alarms and response times occurring within individual video sessions as well as with simple linear regression. Hypothesizing that any alarm fatigue effect would be strongest in the highest alarm patients, and having observed that alarms are distributed very unevenly across patients in both the PICU and ward, we made the decision not to use quartiles, but rather to form clinically meaningful categories. We also hypothesized that nurses might not exhibit alarm fatigue unless they were inundated with alarms. We thus divided the nonactionable alarm counts over the preceding 120 minutes into 3 categories: 0 to 29 alarms to represent a low to average alarm rate exhibited by the bottom $50 \%$ of the patients, 30 to 79 alarms to represent an elevated alarm rate, and $80+$ alarms to represent an extremely high alarm rate exhibited by the top $5 \%$. Because the exposure time was 120 minutes, we conducted the analysis on the alarms occurring after a nurse had been video recorded for at least 120 minutes.

We further evaluated the relationship between nonactionable alarms and nurse response time with KaplanMeier plots by nonactionable alarm count category using the observed response-time data. The KaplanMeier plots compared response time across the nonactionable alarm exposure group, without any statistical modeling. A log-rank test stratified by nurse evaluated whether the distributions of response time in the Kaplan-Meier plots differed across the 3 alarm exposure groups, accounting for within-nurse clustering.

Accelerated failure-time regression based on the Weibull distribution then allowed us to compare response time across each alarm exposure group and provided confidence intervals. Accelerated failure-time models are comparable to Cox models, but emphasize time to event rather than hazards. ${ }^{17,18}$ We determined that the Weibull distribution was suitable by evaluating smoothed hazard and log-hazard plots, the confidence intervals of the shape parameters in the Weibull models that did not include 1 , and by demonstrating that the Weibull model had better fit than an alternative (exponential) model using the likelihood-ratio test $(P<0.0001$ for PICU, $P=0.02$ for ward). Due to the small sample size of nurses and patients, we could not adjust for nurse- or patient-level covariates in the model. When comparing the nonactionable alarm exposure groups in the regression model (0-29 vs 30$79,30-79$ vs $80+$, and $0-29$ vs $80+$ ), we Bonferroni corrected the critical $P$ value for the 3 comparisons, for a critical $P$ value of $0.05 / 3=0.0167$.

\section{Nurse Questionnaire}

At the session's conclusion, nurses completed a questionnaire that included demographics and asked, "Did you respond more quickly to monitor alarms during this study because you knew you were being filmed?" to measure if nurses would report experiencing a Hawthorne-like effect. ${ }^{19-21}$

\section{RESULTS}

We performed 40 sessions among 40 patients and 36 nurses over 210 hours. We performed 20 sessions in children with heart and/or lung failure in the PICU and 20 sessions in children on a general ward. Sessions took place on weekdays between 9:00 AM and 6:00 PM. There were 3 occasions when we filmed 2 patients cared for by the same nurse at the same time.

Nurses were mostly female $(94.4 \%)$ and had between 2 months and 28 years of experience (median, 4.8 years). Patients on the ward ranged from 5 days to 5.4 years old (median, 6 months). Patients in the PICU ranged from 5 months to 16 years old (median, 2.5 years). Among the PICU patients, $14(70 \%)$ were receiving mechanical ventilation only, $3(15 \%)$ were receiving vasopressors only, and $3(15 \%)$ were receiving mechanical ventilation and vasopressors.

We observed 5070 alarms during the 40 sessions. We excluded $108(2.1 \%)$ that occurred at the end of video recording sessions with the nurse absent from the room because the nurse's response could not be determined. Alarms per session ranged from 10 to 1430 (median, 75; interquartile range [IQR], 35-138). We excluded the outlier PICU patient with 1430 alarms in 5 hours from the analysis to avoid the potential for biasing the results. Figure 2 depicts the data flow.

Following the 5 training sessions, research assistants independently reviewed and made preliminary assessments on 4674 alarms; these alarms were all secondarily reviewed by a physician. Using the physician reviewer as the gold standard, the research assistant's sensitivity (assess alarm as actionable when physician also assesses as actionable) was $96.8 \%$ and specificity (assess alarm as nonactionable when physician also assesses as nonactionable) was $96.9 \%$. We had to review 54 of 4674 alarms $(1.2 \%)$ with an additional physician or nurse investigator to achieve consensus.

Characteristics of the 2445 alarms for clinical conditions are shown in Table 1 . Only $12.9 \%$ of alarms in heart- and/or lung-failure patients in the PICU were actionable, and only $1.0 \%$ of alarms in medical patients on a general inpatient ward were actionable.

\section{Overall Response Times for Out-of-Room Alarms}

We first evaluated response times without excluding alarms occurring prior to the 120 -minute mark. Of the 2445 clinical condition alarms, we excluded the 315 noncritical arrhythmia types from analysis of response time because they did not meet our definition of potentially critical alarms. Of the 2130 potentially critical alarms, 1185 (55.6\%) occurred while neither the nurse nor any other clinician was in the patient's room. We proceeded to analyze the response time to these 1185 alarms (307 in the PICU and 878 on the 


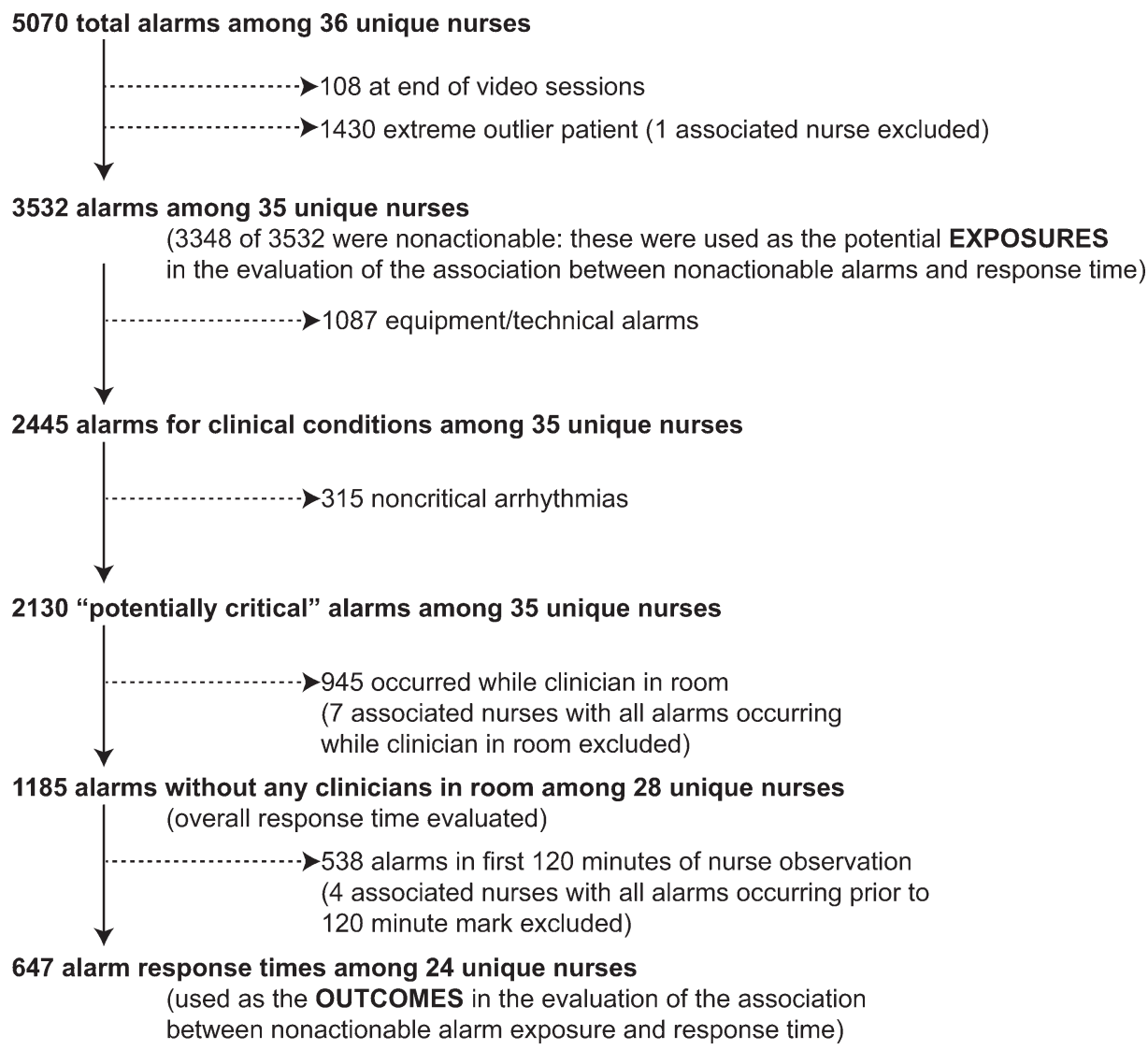

FIG. 2. Flow diagram of alarms used as exposures and outcomes in evaluating the association between nonactionable alarm exposure and response time.

ward). In the PICU, median response time was 3.3 minutes (IQR, 0.8-14.4). On the ward, median response time was 9.8 minutes (IQR, 3.2-22.4).

\section{Response-Time Association With Nonactionable Alarm Exposure}

Next, we analyzed the association between response time to potentially critical alarms that occurred when the nurse was not in the patient's room and the number of nonactionable alarms occurring over the preceding 120-minute window. This required excluding the alarms that occurred in the first 120 minutes of each session, leaving 647 alarms with eligible response times to evaluate the exposure between prior nonactionable alarm exposure and response time: 219 in the PICU and 428 on the ward. Kaplan-Meier plots and tabulated response times demonstrated the incremental relationships between each nonactionable alarm exposure category in the observed data, with the effects most prominent as the Kaplan-Meier plots diverged beyond the median (Figure 3 and Table 2). Excluding the extreme outlier patient had no effect on the results, because 1378 of the 1430 alarms occurred with the nurse present at the bedside, and only 2 of the remaining alarms were potentially critical.

Accelerated failure-time regressions revealed significant incremental increases in the modeled response time as the number of preceding nonactionable alarms increased in both the PICU and ward settings (Table 2).

\section{Hawthorne-like Effects}

Four of the 36 nurses reported that they responded more quickly to monitor alarms because they knew they were being filmed.

\section{DISCUSSION}

Alarm fatigue has recently generated interest among nurses, ${ }^{22}$ physicians, ${ }^{23}$ regulatory bodies, ${ }^{24}$ patient safety organizations, ${ }^{25}$ and even attorneys, ${ }^{26}$ despite a lack of prior evidence linking nonactionable alarm exposure to response time or other adverse patientrelevant outcomes. This study's main findings were that (1) the vast majority of alarms were nonactionable, (2) response time to alarms occurring while the nurse was out of the room increased as the number of nonactionable alarms over the preceding 120 minutes increased. These findings may be explained by alarm fatigue.

Our results build upon the findings of other related studies. The nonactionable alarm proportions we found were similar to other pediatric studies, reporting greater than $90 \%$ nonactionable alarms. ${ }^{1,2}$ One other study has reported a relationship between alarm exposure and response time. In that study, Voepel-Lewis 

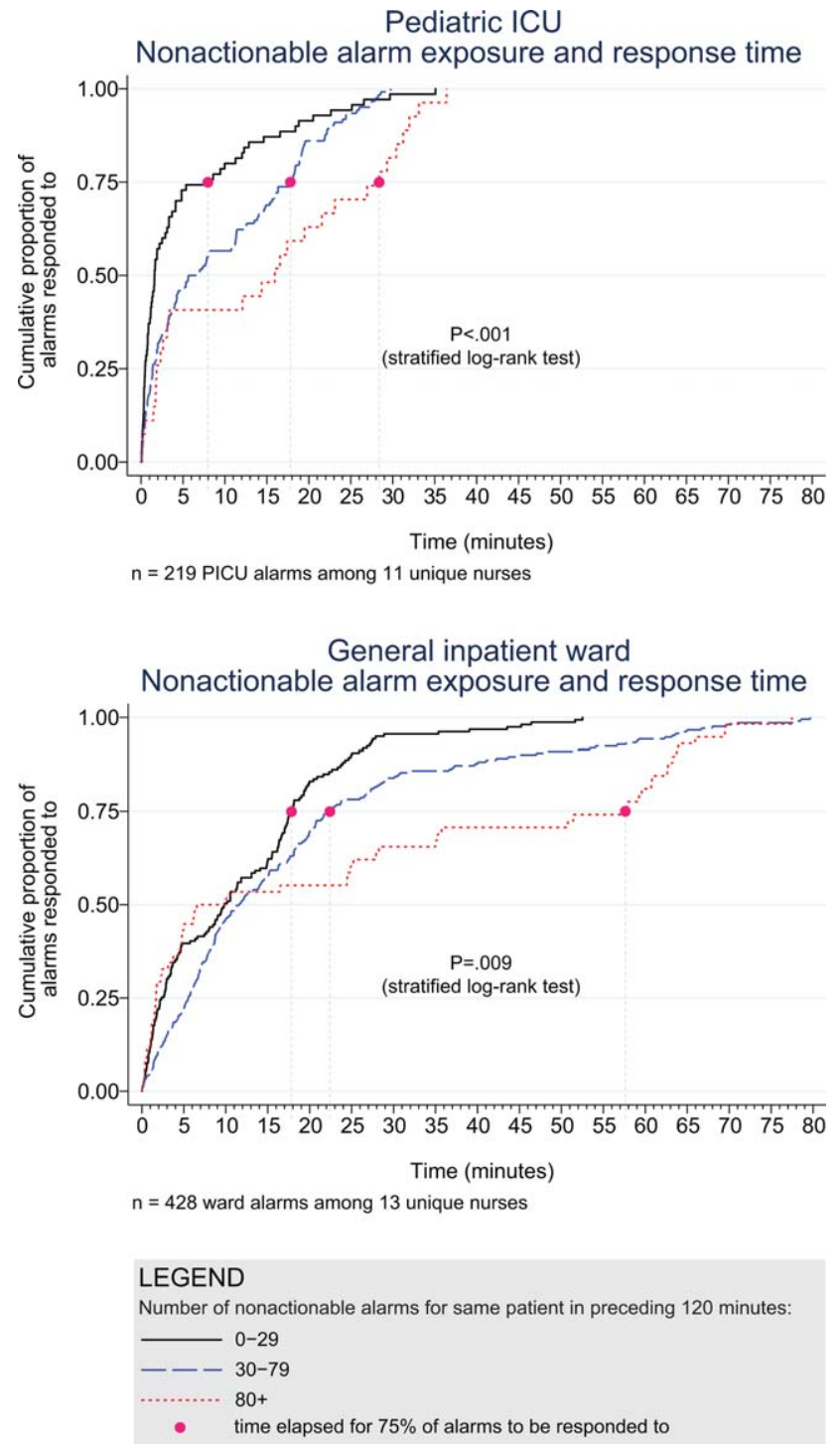

FIG. 3. Kaplan-Meier plots of observed response times for pediatric intensive care unit (PICU) and ward. Abbreviations: ICU, intensive care unit. and colleagues evaluated nurse responses to pulse oximetry desaturation alarms in adult orthopedic surgery patients using time-stamp data from their monitor notification system. ${ }^{27}$ They found that alarm response time was significantly longer for patients in the highest quartile of alarms compared to those in lower quartiles. Our study provides new data suggesting a relationship between nonactionable alarm exposure and nurse response time.

Our study has several limitations. First, as a preliminary study to investigate feasibility and possible association, the sample of patients and nurses was necessarily limited and did not permit adjustment for nurse- or patient-level covariates. A multivariable analysis with a larger sample might provide insight into alternate explanations for these findings other than alarm fatigue, including measures of nurse workload and patient factors (such as age and illness severity). Additional factors that are not as easily measured can also contribute to the complex decision of when and how to respond to alarms. ${ }^{28,29}$ Second, nurses were aware that they were being video recorded as part of a study of nonactionable alarms, although they did not know the specific details of measurement. Although this lack of blinding might lead to a Hawthorne-like effect, our positive results suggest that this effect, if present, did not fully obscure the association. Third, all sessions took place on weekdays during daytime hours, but effects of nonactionable alarms might vary by time and day. Finally, we suspect that when nurses experience critical alarms that require them to intervene and rescue a patient, their response times to that patient's alarms that occur later in their shift will be quicker due to a heightened concern for the alarm being actionable. We were unable to explore that relationship in this analysis because the number of critical alarms requiring intervention was very small. This is a topic of future study.

TABLE 2. Association Between Nonactionable Alarm Exposure in Preceding 120 Minutes and Response Time to Potentially Critical Alarms Based on Observed Data and With Response Time Modeled Using Weibull Accelerated Failure-Time Regression

\begin{tabular}{|c|c|c|c|c|c|c|c|c|}
\hline & \multicolumn{5}{|c|}{ Observed Data } & \multicolumn{3}{|c|}{ Accelerated Failure-Time Model } \\
\hline & \multirow{2}{*}{$\begin{array}{l}\text { Number of Potentially } \\
\text { Critical Alarms }\end{array}$} & \multicolumn{4}{|c|}{$\begin{array}{l}\text { Minutes Elapsed Until This Percentage } \\
\text { of Alarms Was Responded to }\end{array}$} & \multirow{2}{*}{$\begin{array}{l}\text { Modeled Response } \\
\text { Time, min }\end{array}$} & \multirow[b]{2}{*}{$95 \% \mathrm{Cl}, \min$} & \multirow[b]{2}{*}{$P$ Value $^{*}$} \\
\hline & & $50 \%$ (Median) & $75 \%$ & $90 \%$ & $95 \%$ & & & \\
\hline \multicolumn{9}{|l|}{ PICU } \\
\hline 0-29 nonactionable alarms & 70 & 1.6 & 8.0 & 18.6 & 25.1 & 2.8 & $1.9-3.8$ & Reference \\
\hline 30-79 nonactionable alarms & 122 & 6.3 & 17.8 & 22.5 & 26.0 & 5.3 & $4.0-6.7$ & 0.001 (vs 0-29) \\
\hline $80+$ nonactionable alarms & 27 & 16.0 & 28.4 & 32.0 & 33.1 & 8.5 & $4.3-12.7$ & 0.009 (vs 0-29), 0.15 (vs 30-79) \\
\hline \multicolumn{9}{|l|}{ Ward } \\
\hline 0-29 nonactionable alarms & 159 & 9.8 & 17.8 & 25.0 & 28.9 & 7.7 & $6.3-9.1$ & Reference \\
\hline 30-79 nonactionable alarms & 211 & 11.6 & 22.4 & 44.6 & 63.2 & 11.5 & $9.6-13.3$ & 0.001 (vs 0-29) \\
\hline $80+$ nonactionable alarms & 58 & 8.3 & 57.6 & 63.8 & 69.5 & 15.6 & $11.0-20.1$ & 0.001 (vs $0-29$ ), 0.09 (vs 30-79) \\
\hline
\end{tabular}




\section{CONCLUSIONS}

We identified an association between a nurse's prior exposure to nonactionable alarms and response time to future alarms. This finding is consistent with alarm fatigue, but requires further study to more clearly delineate other factors that might confound or modify that relationship.

Disclosures: This project was funded by the Health Research Formula Fund Grant 4100050891 from the Pennsylvania Department of Public Health Commonwealth Universal Research Enhancement Program (awarded to Drs. Keren and Bonafide). Dr. Bonafide is also supported by the National Heart, Lung, and Blood Institute of the National Institutes of Health under award number K23HL116427. The content is solely the responsibility of the authors and does not necessarily represent the official views of the National Institutes of Health. The authors have no financial relationships or conflicts of interest relevant to this article to disclose.

\section{References}

1. Lawless ST. Crying wolf: false alarms in a pediatric intensive care unit. Crit Care Med. 1994;22(6):981-985.

2. Tsien CL, Fackler JC. Poor prognosis for existing monitors in the intensive care unit. Crit Care Med. 1997;25(4):614-619.

3. Biot L, Carry PY, Perdrix JP, Eberhard A, Baconnier P. Clinical evaluation of alarm efficiency in intensive care [in French]. Ann Fr Anesth Reanim. 2000;19:459-466.

4. Borowski M, Siebig S, Wrede C, Imhoff M. Reducing false alarms of intensive care online-monitoring systems: an evaluation of two signal extraction algorithms. Comput Math Methods Med. 2011;2011: 143480.

5. Chambrin MC, Ravaux P, Calvelo-Aros D, Jaborska A, Chopin C, Boniface B. Multicentric study of monitoring alarms in the adult intensive care unit (ICU): a descriptive analysis. Intensive Care Med. 1999; 25:1360-1366.

6. Görges M, Markewitz BA, Westenskow DR. Improving alarm performance in the medical intensive care unit using delays and clinical context. Anesth Analg. 2009;108:1546-1552.

7. Graham KC, Cvach M. Monitor alarm fatigue: standardizing use of physiological monitors and decreasing nuisance alarms. Am J Crit Care. 2010;19:28-34.

8. Siebig S, Kuhls S, Imhoff M, Gather U, Scholmerich J, Wrede CE. Intensive care unit alarms-how many do we need? Crit Care Med. 2010;38:451-456.

9. Getty DJ, Swets JA, Rickett RM, Gonthier D. System operator response to warnings of danger: a laboratory investigation of the effects of the predictive value of a warning on human response time. J Exp Psychol Appl. 1995;1:19-33.

10. Bliss JP, Gilson RD, Deaton JE. Human probability matching behaviour in response to alarms of varying reliability. Ergonomics. 1995; 38:2300-2312.
11. The Joint Commission. Sentinel event alert: medical device alarm safety in hospitals. 2013. Available at: http://www.jointcommission. org/sea_issue_50/. Accessed October 9, 2014.

12. Mitka M. Joint commission warns of alarm fatigue: multitude of alarms from monitoring devices problematic. JAMA. 2013;309(22): 2315-2316.

13. Cvach M. Monitor alarm fatigue: an integrative review. Biomed Instrum Technol. 2012;46(4):268-277.

14. NIH Certificates of Confidentiality Kiosk. Available at: http:/grants. nih.gov/grants/policy/coc/. Accessed April 21, 2014.

15. Bonafide CP, Zander M, Graham CS, et al. Video methods for evaluating physiologic monitor alarms and alarm responses. Biomed Instrum Technol. 2014;48(3):220-230.

16. Harris PA, Taylor R, Thielke R, Payne J, Gonzalez N, Conde JG. Research electronic data capture (REDCap)—a metadata-driven methodology and workflow process for providing translational research informatics support. J Biomed Inf. 2009;42:377-381.

17. Collett D. Accelerated failure time and other parametric models. In: Modelling Survival Data in Medical Research. 2nd ed. Boca Raton, FL: Chapman \& Hall/CRC Press; 2003:197-229.

18. Cleves M, Gould W, Gutierrez RG, Marchenko YV. Parametric models. In: An Introduction to Survival Analysis Using Stata, 3rd ed. College Station, TX: Stata Press; 2010:229-244.

19. Roethlisberger FJ, Dickson WJ. Management and the Worker. Cambridge, MA: Harvard University Press; 1939.

20. Parsons HM. What happened at Hawthorne? Science. 1974; 183(4128):922-932.

21. Ballermann M, Shaw N, Mayes D, Gibney RN, Westbrook J. Validation of the Work Observation Method By Activity Timing (WOMBAT) method of conducting time-motion observations in critical care settings: an observational study. BMC Med Inf Decis Mak. 2011;11: 32.

22. Sendelbach S, Funk M. Alarm fatigue: a patient safety concern. AACN Adv Crit Care. 2013;24(4):378-386.

23. Chopra V, McMahon LF Jr. Redesigning hospital alarms for patient safety: alarmed and potentially dangerous. JAMA. 2014;311(12): $1199-1200$

24. The Joint Commission. The Joint Commission announces 2014 National Patient Safety Goal. Jt Comm Perspect. 2013;33:1-4.

25. Top 10 health technology hazards for 2014. Health Devices. 2013; 42(11):354-380.

26. My Philly Lawyer. Medical malpractice: alarm fatigue threatens patient safety. 2014. Available at: http://www.myphillylawyer.com/ Resources/Legal-Articles/Medical-Malpractice-Alarm-Fatigue-Threatens-Patient-Safety.shtml. Accessed April 4, 2014.

27. Voepel-Lewis T, Parker ML, Burke CN, et al. Pulse oximetry desaturation alarms on a general postoperative adult unit: a prospective observational study of nurse response time. Int J Nurs Stud. 2013; 50(10):1351-1358.

28. Gazarian PK, Carrier N, Cohen R, Schram H, Shiromani S. A description of nurses' decision-making in managing electrocardiographic monitor alarms [published online ahead of print May 10, 2014]. J Clin Nurs. doi:10.1111/jocn.12625.

29. Gazarian PK. Nurses' response to frequency and types of electrocardiography alarms in a non-critical care setting: a descriptive study. Int J Nurs Stud. 2014;51(2):190-197. 